\title{
Photodegradable organic pollutants from wastewater
}

\author{
Oana Stoian ${ }^{1}$, Cristina Ileana Covaliu ${ }^{1, *}$, Gigel Paraschiv ${ }^{1}$, Mihai Nita-Lazar ${ }^{2}$, and \\ Ioana- Corina $\mathrm{Moga}^{3}$ \\ ${ }^{1}$ Politehnica University of Bucharest, 313 Splaiul Independentei, 060042, Bucharest, Romania \\ ${ }^{2}$ Research and Development Institute for Industrial Ecology - ECOIND, 71-73 Drumul Podu \\ Dambovitei Str., 060652, Bucharest, Romania \\ ${ }^{3}$ Dfr Systems SRL, Drumul Taberei 46, Bucharest, Romania
}

\begin{abstract}
Nowadays, environmental pollution is constantly increasing, and depollution is the most important challenge of the $21^{\text {st }}$ century. This paper is a review of organic pollutants in wastewater that can be removed by the photocatalysis process. Also are presented specialized studies that demonstrate the removal of organic pollutants from wastewater and the experimental conditions necessary to obtain high treatment efficiencies. The important classes of pollutants in wastewater are hydrocarbons, halo compounds, carbonyl derivatives, alcohols, phenols, pesticides, surfactants, nitrogen containing compounds and dyes, and represent a major source of water pollution. These organic pollutants are photodegradable and can be easily removed by the photocatalysis process.
\end{abstract}

\section{Introduction}

Toxic organic pollutants from domestic use or from the activity of various industries are a major environmental problem through water pollution, and their remediation is a considerable concern [1]. A large number of organic pollutants such as dyes, pesticides or pharmaceuticals are released daily into many wastewaters, which are then discharged into natural waters and accumulate in the aquatic environment [2-6]. Pollution comes from domestic, industrial and agricultural activities and cannot be stopped by conventional water treatment methods. Thus, synthetic organic substances have been detected in small quantities in lakes, rivers, oceans or drinking water. In order to remove organic pollutants from wastewater, safe, efficient, economical and simple technologies are developed to avoid toxic consequences on human health [7]. Organic pollutants removed / degraded from wastewater by the photocatalysis process are described in the following subchapters.

Factors that may influence the photocatalysis process used to degrade wastewater pollutants are pollutant nature, concentration and the existence of other compounds in wastewater, the amount of photocatalysts added, their size and structure, water $\mathrm{pH}$ and temperature, light intensity and irradiation time [8]. Also, the reactor design, the number of UV lamps and their characteristics influence the photocatalysis process. High concentration

\footnotetext{
* Corresponding author: cristina_covaliu@yahoo.com
} 
of pollutants in wastewater can saturate the surface of the photocatalyst and thus, at some point, can reduce photonic efficiency or deactivate the photocatalyst [9]. As mentioned above, the photocatalysis process also depends on the nature of the pollutants. If the pollutants are compatible with the photocatalyst used to adhere efficiently to its surface, the process will be more efficient and faster in order to remove these pollutants from the wastewater [8]. The degradation of the pollutant increases with the increase of the amount of catalyst added in the wastewater. This phenomenon is explained by the fact that the addition of a larger amount of photocatalyst leads to an increase in the number of active sites that are needed to remove pollutants from wastewater [10]. The surface morphology is a major factor that influences the photocatalysis process due to the existence of a direct relationship between the pollutant and the photocatalyst surface [11]. Changing the $\mathrm{pH}$ of the wastewater will change the potential of photocatalytic reactions and will influence the reaction rate. Depending on the acidic or alkaline aqueous medium, the surface of the photocatalyst can be protonated or deprotonated [12]. The increase of the reaction temperature will lead to an increased photocatalytic activity. However, a temperature higher than $80^{\circ} \mathrm{C}$ disadvantages the removal of pollutants using photocatalysts due to the recombination of charge carriers. The temperature range considered adequate in the photocatalysis process is $20-80^{\circ} \mathrm{C}$ [13].

\subsection{Hydrocarbons}

Water pollution with hydrocarbons has negative effects on all life forms, forming a toxic film at the water surface, thus reducing the power of the sun to penetrate. In this case, the aquatic plants photosynthetic activity will decrease. The main sources of water pollution with hydrocarbons are represented by the oil platforms as well as the oil transport ships, by accidental pollution or by the discharge into the sea of the washing water of the ship tanks. By forming water-impermeable film due to crude oil and petroleum products, the exchange of oxygen between the environment and water can no longer be achieved, causing damage to animals, plants and humans [14].

\subsection{Halo compounds}

Halo-organic compounds are pollutants that are used as dyes, agrochemicals, solvents, drugs, etc. These compounds are consumed by humans and animals by accumulating in aquatic plants and animals [15].

In order to transform halo-organics into other non-polluting compounds, treatment methods such as incineration, extraction, electrochemical treatment, chemical degradation, sonochemical destruction and photochemical process are applied. The latter was found to be the most useful method used in the degradation process of halo compounds in wastewater [16-18].

\subsection{Carbonyl derivatives}

Carbonyl compounds are recognized as carboxylic acids, ketones, aldehydes and quinones [15]. Table 1 shows carbonyl derivatives organic pollutants removed from wastewater by photocatalysis using photocatalysts containing $\mathrm{TiO}_{2}$. Also, the experimental conditions and the treatment efficiencies are presented in this table. 
Table 1. Examples of carbonyl derivatives removal from wastewater, conditions and efficiencies

\begin{tabular}{|c|c|c|c|c|c|}
\hline $\begin{array}{c}\text { Photocatalytic } \\
\text { material }\end{array}$ & Pollutant & $\begin{array}{c}\text { Initial } \\
\text { concentration } \\
{[\mathrm{mg} / \mathrm{L}]}\end{array}$ & $\begin{array}{c}\text { Time } \\
{[\mathrm{min}]}\end{array}$ & $\begin{array}{c}\text { Efficiency } \\
{[\%]}\end{array}$ & Ref. \\
\hline $\mathrm{Pt}-\mathrm{TiO}_{2}$ & Oxalic acid & 1,98 & 60 & $100,00 \%$ & {$[19]$} \\
\hline $\mathrm{TiO}_{2}$ & Salicylic acid & - & 90 & $17,00 \%$ & {$[20]$} \\
\hline $\begin{array}{c}\text { Polyacrylanitrile } \\
-\mathrm{TiO}_{2}+\mathrm{H}_{2} \mathrm{O}_{2}\end{array}$ & Formaldehyde & 90,00 & 250 & $\approx 86,00 \%$ & {$[21]$} \\
\hline
\end{tabular}

\subsection{Alcohols}

The alcohol industry is considered a major source of environmental pollution. After proper treatment of these waters polluted with alcohol, they can be used for fertilized irrigation [22]. Table 2 shows alcohols as organic pollutants in wastewater that are removed by the photocatalysis process using photocatalysts containing $\mathrm{TiO}_{2}$. Also, the initial pollutant concentrations, the required treatment time and the efficiencies are presented in this table.

Table 2. Removal of alcohols from wastewater, conditions and efficiencies

\begin{tabular}{|c|c|c|c|c|c|}
\hline $\begin{array}{c}\text { Photocatalytic } \\
\text { material }\end{array}$ & Pollutant & $\begin{array}{c}\text { Initial concentration } \\
{[\mathrm{mg} / \mathrm{L}]}\end{array}$ & $\begin{array}{l}\text { Time } \\
{[\mathrm{min}]}\end{array}$ & $\begin{array}{c}\text { Efficiency } \\
{[\%]}\end{array}$ & Ref. \\
\hline $\mathrm{TiO}_{2}$ Degussa 25 & n-butanol & 580,00 & 30 & $99,00 \%$ & [23] \\
\hline \multirow{2}{*}{$\begin{array}{c}\text { Photocatalytic } \\
\text { media containing } \\
\mathrm{TiO}_{2}\end{array}$} & $\begin{array}{l}\text { Isopropyl } \\
\text { alcohol }\end{array}$ & \multirow{2}{*}{1,00} & \multirow{2}{*}{120} & $97,20 \%$ & \multirow{2}{*}{ [24] } \\
\hline & Ethanol & & & $99,00 \%$ & \\
\hline
\end{tabular}

\subsection{Phenols}

Phenolic compounds from wastewater come from natural, domestic, industrial and agricultural activities. Following the decomposition or degradation of natural organic matter in water, the disposal of household and industrial waste in water or runoff from agricultural land, phenolic compounds may occur in wastewater [25]. They are aromatic compounds, having attached on the aromatic ring one or more hydroxyl groups. The discharge of toxic phenolic compounds into lakes, rivers or the sea comes from the oil industry, the manufacture of phenolic resins, plastics, wood products, textiles, pharmaceuticals, detergents, cellulose, pesticide synthesis and coking operations [26, 27]. Also, in aquatic environments can be generated phenolic pollutants, from the secretion of algae, flavonoids, hydrolysable tannins and lignin transformation [28]. Due to their corrosive nature, phenols can cause irritation to the eyes, skin and airways if they are absorbed by the lungs [29]. Table 3 shows the removal of phenols from wastewater by using photocatalysts containing $\mathrm{TiO}_{2}$. 
Table 3. Examples of phenols removal from wastewater

\begin{tabular}{|c|c|c|c|c|c|}
\hline $\begin{array}{c}\text { Photocatalytic } \\
\text { material }\end{array}$ & Pollutant & $\begin{array}{c}\text { Initial } \\
\text { concentration } \\
{[\mathrm{mg} / \mathrm{L}]}\end{array}$ & $\begin{array}{c}\text { Time } \\
{[\mathrm{min}]}\end{array}$ & $\begin{array}{c}\text { Efficiency } \\
{[\%]}\end{array}$ & Ref. \\
\hline $\mathrm{TiO}_{2}$ & Phenol & 30,00 & 300 & $50,00 \%$ & {$[30]$} \\
\hline $\begin{array}{c}\mathrm{TiO}_{2} \text { supported } \\
\text { on ZSM-5 }\end{array}$ & Phenol & 90,00 & 30 & $>90,00 \%$ & {$[31]$} \\
\hline $\begin{array}{c}\mathrm{TiO}_{2} \mathrm{P} 25 \\
\text { Degussa }\end{array}$ & $\begin{array}{c}3- \\
\text { aminophenol }\end{array}$ & 10,91 & 160 & $52,80 \%$ & {$[32]$} \\
\hline
\end{tabular}

\subsection{Pesticides}

Pesticides are an important class of water pollutants. Pesticides such as betahexachlorocyclohexane organochemicals, organophosphorus (representing a group of insecticides) cypermethrin, dicofol, etc. are commonly used [15].

The efficient methods used to degrade pesticides are mechanical-chemical destruction, precipitation, microbial degradation, thermal degradation, adsorption on activated carbon and photocatalytic degradation. Due to the long contact time required and the high cost, some methods are not so suitable for pesticide degradation. An easy-to-operate, fast and low-cost process is required. Specialized studies show that the technology successfully used to remove or degrade pesticides is photocatalytic oxidation [15]. Table 4 shows that pesticides are efficiently removed from wastewater through the photocatalysis process by using titanium dioxide as a catalyst.

Table 4. Degradation of pesticides from wastewater

\begin{tabular}{|c|c|c|c|c|c|}
\hline $\begin{array}{c}\text { Photocatalytic } \\
\text { material }\end{array}$ & Pollutant & $\begin{array}{c}\text { Initial } \\
\text { concentration } \\
{[\mathrm{mg} / \mathrm{L}]}\end{array}$ & $\begin{array}{l}\text { Time } \\
\text { [min] }\end{array}$ & $\begin{array}{c}\text { Efficiency } \\
{[\%]}\end{array}$ & Ref. \\
\hline $\begin{array}{c}\mathrm{TiO}_{2} \text { Degussa } \mathrm{P}- \\
25\end{array}$ & $\begin{array}{l}\text { Pirimiphos - } \\
\text { methyl }\end{array}$ & 9,70 & 40 & $100,00 \%$ & [33] \\
\hline \multirow{3}{*}{ Anatase $\mathrm{TiO}_{2}$} & Lindane & \multirow{4}{*}{40,00} & & $77,00 \%$ & \multirow{4}{*}{ [34] } \\
\hline & Methoxychlor & & & $>99,00 \%$ & \\
\hline & \multirow{2}{*}{$\mathrm{p}, \mathrm{p}^{\prime}-\mathrm{DDT}$} & & 120 & $95,00 \%$ & \\
\hline Rutile $\mathrm{TiO}_{2}$ & & & 150 & $85,00 \%$ & \\
\hline
\end{tabular}

DDT - dichlorodiphenyltrichloroethane

\subsection{Surfactants}

Surfactants are composed of a water-soluble hydrophilic group and an oil-soluble hydrophobic group, two groups having the opposite nature. Surfactants are used in the fields of research, technology, cosmetics, pharmacy, textiles, biotechnology or agriculture on a large scale due to the fact that they possess favourable physico-chemical properties. 
Due to the foaming tendency and the low oxygenation potential, surfactants pose a great threat to the aquatic environment. Types of surfactants can be ionic and nonionic. Ionic surfactants are cationic, anionic and amphoteric surfactants [15]. Table 5 shows the water treatment efficiency with surfactant content through the photocatalysis process.

Table 5. Surfactants degradation from wastewater

\begin{tabular}{|c|c|c|c|c|c|}
\hline \multirow{2}{*}{$\begin{array}{c}\text { Photocatalytic } \\
\text { material }\end{array}$} & Pollutant & $\begin{array}{c}\text { Initial } \\
\text { concentration } \\
{[\mathrm{mg} / \mathrm{L}]}\end{array}$ & $\begin{array}{c}\text { Time } \\
{[\mathrm{min}]}\end{array}$ & $\begin{array}{c}\text { Efficiency } \\
{[\%]}\end{array}$ & Ref. \\
\hline \multirow{2}{*}{$\mathrm{TiO}_{2}$} & $\mathrm{SLS}, \mathrm{SDDBS}$ & 50,00 & 60 & $80,00 \%$ & {$[35]$} \\
\cline { 2 - 5 } & $\mathrm{DBS}$ & 20,00 & 120 & $>80,00 \%$ & \\
\cline { 2 - 2 } & $\mathrm{SDS}$ & & & $>90,00 \%$ & {$[36]$} \\
\hline $\mathrm{TiO}_{2}-\mathrm{Cu}_{2} \mathrm{O}$ & $\mathrm{DBS}$ & 100,00 & - & $97,30 \%$ & {$[37]$} \\
\hline
\end{tabular}

SLS - sodium lauryl sulphate,

SDDBS - sodium dodecylbenzenesulphonate,

SDS - sodium dodecyl sulphate,

DBS - dodecylbenzenesulfonate.

\subsection{Nitrogen containing compounds}

Nitrogen-containing compounds include proteins, amino acids, pesticides, herbicides, drugs, dyes and more. Due to their toxicity and stability in the environment, they can pose a serious threat [15]. Nitrobenzene, as an example of a nitrogen containing compound, is presented in the Table 6 as an organic pollutant that can be degraded by photocatalysis, obtaining high water treatment efficiencies.

Table 6. Nitrobenzene degradation from wastewater

\begin{tabular}{|c|c|c|c|c|c|}
\hline $\begin{array}{c}\text { Photocatalytic } \\
\text { material }\end{array}$ & Pollutant & $\begin{array}{c}\text { Initial } \\
\text { concentration } \\
{[\mathrm{mg} / \mathrm{L}]}\end{array}$ & $\begin{array}{l}\text { Time } \\
\text { [min] }\end{array}$ & $\begin{array}{c}\text { Efficiency } \\
{[\%]}\end{array}$ & Ref. \\
\hline $\begin{array}{c}\text { Nanocrystalline } \\
\mathrm{TiO}_{2}\end{array}$ & \multirow{4}{*}{ Nitrobenzene } & \multirow{2}{*}{50,00} & 180 & 100,00 & \multirow{2}{*}{38} \\
\hline $\begin{array}{c}\text { P-25 Degussa } \\
\mathrm{TiO}_{2}\end{array}$ & & & & 100,00 & \\
\hline $\mathrm{TiO}_{2}$ nanotubes & & \multirow{2}{*}{40,00} & \multirow{2}{*}{240} & 100,00 & \multirow{2}{*}{ [39] } \\
\hline $\mathrm{TiO}_{2}$ powder & & & & 85,00 & \\
\hline
\end{tabular}

\subsection{Dyes}

Dyes represent the class of pollutants that is visually observable due to the coloration of the water. Because of this, but also because of their toxicity, dyes have received a lot of 
attention [40]. 10,000 dyes are commercially available and are used in the production of textiles, paints, plastics, paper, cosmetics, rubber or tanneries [41]. Dyes are a major danger to living organisms in water and negative impacts on humans are represented by symptoms such as nausea, abdominal discomfort, vomiting and irritation [40]. Table 7 shows the removal of dyes from wastewater by using photocatalysts containing $\mathrm{TiO}_{2}$. Also, the initial pollutant concentrations, the required treatment time and the efficiencies are presented in this table.

Table 7. Dyes degradation from wastewater

\begin{tabular}{|c|c|c|c|c|c|}
\hline $\begin{array}{c}\text { Photocatalytic } \\
\text { material }\end{array}$ & Pollutant & $\begin{array}{c}\text { Initial } \\
\text { concentration } \\
{[\mathrm{mg} / \mathrm{L}]} \\
\end{array}$ & $\begin{array}{l}\text { Time } \\
\text { [min] }\end{array}$ & $\begin{array}{c}\text { Efficiency } \\
{[\%]}\end{array}$ & Ref. \\
\hline \multirow{2}{*}{$\mathrm{Nano}-\mathrm{SrTiO}_{3}$} & Direct Green & \multirow{2}{*}{20,00} & \multirow{2}{*}{180} & $\approx 84,00$ & \multirow{2}{*}[42]{} \\
\hline & $\begin{array}{l}\text { Reactive } \\
\text { Orange }\end{array}$ & & & $\approx 88,00$ & \\
\hline \multirow{3}{*}{$\begin{array}{c}\mathrm{CA}-\mathrm{CNT} / \mathrm{TiO}_{2} \\
-\mathrm{NH}_{2}\end{array}$} & \multirow{3}{*}{$\begin{array}{l}\text { Methylene } \\
\text { blue }\end{array}$} & 10 & \multirow{3}{*}{300} & 100,00 & \multirow{3}{*}{ [43] } \\
\hline & & 30 & & 80,00 & \\
\hline & & 50 & & 70,00 & \\
\hline \multirow{3}{*}{$\begin{array}{c}\mathrm{CA}-\mathrm{CNT} / \mathrm{TiO}_{2} \\
-\mathrm{NH}_{2}\end{array}$} & \multirow{3}{*}{ Indigo carmin } & 10 & \multirow{3}{*}{180} & 100,00 & \multirow{3}{*}[43]{} \\
\hline & & 30 & & 90,00 & \\
\hline & & 50 & & 80,00 & \\
\hline \multirow{2}{*}{$\mathrm{Fe}_{2} \mathrm{O}_{3} / \mathrm{TiO}_{2}$} & Methyl orange & \multirow{2}{*}{30} & \multirow{2}{*}{210} & 61,50 & \multirow{2}{*}{ [44] } \\
\hline & Congo red & & & 46,80 & \\
\hline
\end{tabular}

CA - cellulose acetate

\section{Conclusions}

The heterogeneous photocatalysis process is found in Advanced Oxidation Processes (AOP) and has great potential for degradation of wastewater pollutants. In this review were presented the organic pollutants that can be degraded by the photocatalysis process. Applying this process were obtained high treatment efficiencies for the removal / degradation of organic pollutants from wastewater. From all the catalysts titanium dioxide plays an important role. The application of the photocatalysis process has advantages such as the degradation of organic pollutants or their transformation into non-toxic chemicals by using $\mathrm{TiO}_{2}$-based catalysts. Using this process does not generate other pollutants or the necessary energy resource comes largely from natural solar energy which is unlimited. 
The photocatalysis process could have some limitations represented by the possibility of not reaching a maximum treatment efficiency, the difficulty of distributing the photocatalyst particles uniformly, the difficulty of recovering nanoparticles from the system, agglomeration of nanoparticles or inefficient use of visible light. However, these limitations can be remedied by improving the process or photocatalysts used.

This work was supported by a grant of the Romanian Ministry of Education and Research, CCDI UEFISCDI, project number PN-III-P2-2.1-PTE-2019-0628, within PNCDI III.

\section{References}

1. J. Zhao, C. Chen, W. Ma, Catalysis, 35, 3-4 (2005)

2. J. P. Bound, N. Voulvoulis, Chemosphere 56, 1143 (2004)

3. S. K. Khetan, T. J. Collins, Chem. Rev. 107, 2319 (2007)

4. K. Kümmerer, Chemosphere 75, 417 (2009)

5. C. A. Martínez-Huitle, E. Brillas, Appl. Catal., 87, 105 (2009)

6. Y. Luo, L. Xu, M. Rysz, Y. Wang, H. Zhang, P. J. J. Alvarez, Environ. Sci. Technol. 45, 1827 (2011)

7. E. Brillas, J. Braz, Chem. Soc., 25, 3, 393-417 (2014)

8. A. Kumar, G. Pandey, Material Sci. \& Eng. Int. J., 1, 3, 106-114 (2017)

9. A. Sobczynski, A. Dobosz, Pol. J. Environ. Stud., 10, 4, 195-205 (2001)

10. H. M. Coleman, V. Vimonses, G. Leslie, et al., J. Hazard. Mater., 146, 3, 496-501 (2007)

11. C. Kormann, D. W. Bahnemann, M. R. Hoffman, Environ. Sci. Technol., 22, 7, 798806 (1988)

12. R. J. Davis, J. L. Gainer, G. O. Neal, et al. Water Environ. Res., 66, 1, 50-53 (1994)

13. G. Mamba, M. A. Mamo, X. Y. Mbianda, et al., Ind. Eng. Chem. Res., 53, 37, 1432914338 (2014)

14. M. Srivastava, A. Srivastava, A. Yadav, V. Rawat, Hydrocarbon Pollution and Its Effect on the Environment (2019)

15. R. Ameta, S. Benjamin, A. Ameta, S. C. Ameta, Mat. Sci. Forum, 734, 247-272 (2013)

16. D. F. Ollis, Environ. Sci. Technol., 19, 480-484 (1985)

17. D. Cesareo, D. A. Di, S. Marchini, L. Passerini, M. L. Tosato, Homo.-Hetero. Photocatal., 174, 593-627 (1986)

18. D. F. Ollis, E. Pelizzetti, N. Serpone, Photocatalyzed destruction of water contaminants, Environ. Sci. Technol., 25, 1522-1529 (1991)

19. Y. Li, G. Lu, S. Li, Applied Catalysis A: General, 214, 179-185 (2001)

20. V. Sukharev, A. Wold, Y-M. Gao, K. Dwight, J. Solid State Chem., 119, 339-343 (1995)

21. R. Bonora, C. Boaretti, L. Campea, M. Roso, A. Martucci, M. Modesti, A. Lorenzetti, Nanomat., 10, 148 (2020)

22. P. Chowdhary, R. N. Bharagava, Emerging and Eco-Friendly Approaches for Waste Management (2018)

23. J. Kirchnerova, M.-L. Herrera Cohen, C. Guy, D. Klvana, Appl. Cat. A: General, 282, 321-332 (2005)

24. J. Chung, S. Chung, G. Lee, Y-W. Lee, Int. J. Environ. Res. Public Health, 17, 4203 (2020)

25. W. Anku, M. Mamo, P. Govender, Phenolic Compounds in Water: Sources, Reactivity, Toxicity and Treatment Methods (2017)

26. S. Das, N. Kuppanan, V.A. Channashettar, B. Lal,. Springer, 165-177 (2018) 
27. L.G.C. Villegas, N. Mashhadi, M. Chen, D. Mukherjee, K.E. Taylor, N. Biswas, J. Current Poll. Rep., 2, 3, 157-167 (2016)

28. N.F. Zainudin, A.Z. Abdullah, A.R. Mohamed, J. Hazard Mater., 174, 1-3, 299-306 (2010)

29. R. Rathna, E. Nakkeeran, Springer, 253-276 (2018)

30. A. Nickheslat, M. M. Amin, H. Izanloo, A. Fatehizadeh, S. M. Mousavi, J. Environ. Public Health, 2013 (2013)

31. J. Chen, L. Eberlein, C. H. Langford, J. Photochem. Photobiol. A: Chem., 148, 183189 (2002)

32. N. San, A. Hatipoglu, G. Koçtürk, Z. Çınar, J. Photochem. Photobiol. A: Chem., 139, 225-232 (2001)

33. J. M. Herrmann, C. Guillard, M. Arguello, A. Agüera, A. Tejedor, L. Piedra, A. Fernández-Alba, Cat. Today, 54, 353-367 (1999)

34. A. Zaleska, J. Hupka, M. Wiergowski, M. Biziuk, J. Photochem. Photobiol. A: Chem., 135, 213-220 (2000)

35. C. Lizama; C. Bravo; C. Caneo, M. Ollino, Environ. Tech., 26, 909-914 (2010)

36. A. M. Amat, A. Arques, M. A. Miranda, S. Segu, Solar Energy, 77, 559-566 (2004)

37. C. Han, Z. Li, J. Shen, J. Haz. Mat., 168, 215-219 (2009)

38. R. J. Tayade, R. G. Kulkarni, R. V. Jasra, Ind. Eng. Chem. Res., 45, 922-927 (2006)

39. R. J. Tayade, D. L. Key, Mat. Sci. Forum, 657, 62-74 (2010)

40. S. Shakoor, A. Nasar,, J. Taiwan Inst. Chem. Eng., 66, 154-163 (2016)

41. N., Peng, D., Hu, J., Zeng, Y., Li, L., Liang, C., Chang, J ACS Sustainable Chem. Eng., 4, 12, 7217-7224 (2016)

42. L. Karimi, S. Zohoori, M. E. Yazdanshenas, J. Saudi Chem. Society, 18, 581-588 (2014)

43. A. Salama, A. Mohamed, N. M. Aboamera, T. A. Osman, A. Khattab, App. Nanosci., 8, 155-161(2018)

44. M. Nasirian, C. F. Bustillo-Lecompte, M. Mehrvar, J. Environ. Management, 196, 487$498(2017)$ 